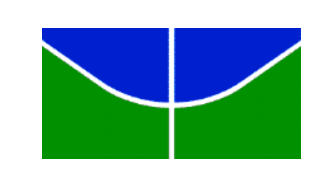

UNIVERSIDADE DE BRASÍLIA

Faculdade de Agronomia e Medicina Veterinária

Programa de Pós-Graduação em Saúde Animal

\title{
AVALIAÇÃO DOS GRADIENTES DE TEMPERATURA EM GATOS HÍGIDOS
}

KAMILA SANTOS DE MORAIS

\author{
DISSERTAÇÃO DE MESTRADO \\ EM SAÚDE ANIMAL
}

Brasília/DF

Fevereiro/2016 


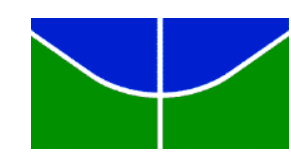

UNIVERSIDADE DE BRASÍLIA

Faculdade de Agronomia e Medicina Veterinária

Programa de Pós-Graduação em Saúde Animal

AVALIAÇÃO DOS GRADIENTES DE TEMPERATURA EM GATOS HÍGIDOS

KAMILA SANTOS DE MORAIS

ORIENTADORA: Profa. GLÁUCIA BUENO PEREIRA NETO

DISSERTAÇÃO DE MESTRADO EM SAÚDE ANIMAL ÁREA DE CONCENTRAÇÃO: CLÍNICA MÉDICA E CIRÚRGICA ANIMAL LINHA DE PESQUISA: MÉTODOS DE DIAGNÓSTICO E TRATAMENTO DE AFECÇÕES DOS ANIMAIS DOMÉSTICOS E SILVESTRES

PUBLICAÇÃ̃: 126

Brasília/DF

Fevereiro/2016 
UNIVERSIDADE DE BRASÍLIA

AVALIAÇÃO DOS GRADIENTES DE TEMPERATURA EM GATOS HÍGIDOS

KAMILA SANTOS DE MORAIS

DISSERTAÇÃO DE MESTRADO SUBMETIDA AO PROGRAMA DE PÓSGRADUAÇÃO EM SAÚDE ANIMAL, COMO PARTE DOS REQUISITOS NECESSÁRIOS À OBTENÇÃO DO GRAU DE MESTRE EM SAÚDE ANIMAL.

APROVADA POR:

GBonibi.

GLÁUCIA BUENO PEREIRA NETO, Professora Doutora (FAV/UnB), Orientadora

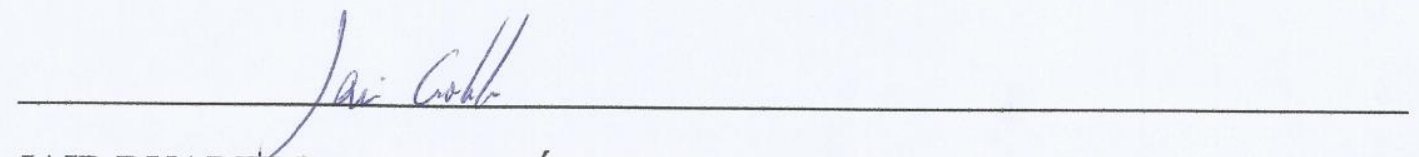

JAIR DUARTE DA COSTA JÚNIOR, Professor Doutor (FAV/UnB)

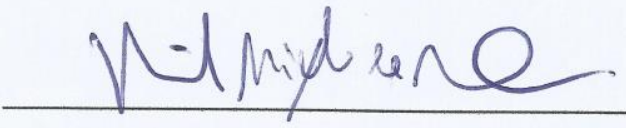

RICARDO MIYASAKA DE ALMEIDA, Professor Doutor (FAV/UnB)

BRASÍLIA/DF, 29 DE FEVEIRO DE 2016. 


\section{REFERÊNCIA BIBLIOGRÁFICA E CATALOGAÇÃO}

MORAIS, K. S. Avaliação dos gradientes de temperatura em gatos hígidos. Brasília: Faculdade de Agronomia e Medicina Veterinária, Universidade de Brasília, 2016, 42p. Dissertação de Mestrado.

Documento formal, autorizando reprodução desta dissertação de mestrado para empréstimo ou comercialização, exclusivamente para fins acadêmicos, foi passado pelo autor à Universidade de Brasília e acha-se arquivado na Secretaria do Programa. O autor reserva para si os outros direitos autorais, de publicação. Nenhuma parte desta dissertação de mestrado pode ser reproduzida sem a autorização por escrito do autor. Citações são estimuladas, desde que citada a fonte.

Morais, Kamila Santos de

Avaliação dos gradientes de temperatura em gatos hígidos /Kamila Santos de Morais Orientação de Gláucia Bueno Pereira Neto

Brasília, 2016. 42 p. : il.

Dissertação de Mestrado (M) - Universidade de Brasília/Faculdade de Agronomia e Medicina Veterinária, 2016.

1. Perfusão periférica 2. Gradientes de Temperatura Periférica 3. Lactato I. MORAIS. K.S. II. Título 


\section{AGRADECIMENTOS}

Gostaria de agradecer primeiramente a Deus, por iluminar o meu caminho nesta jornada e sempre ter me abençoando com muita fé e perseverança para enfrentar todos os desafios.

À minha família, por ser meu alicerce, mostrando os diferentes pontos de vista para que as decisões fossem as mais coerentes e justas.

Ao Rodrygo, meu grande companheiro e melhor amigo, sempre me ajudando em todos os momentos.

Ao Hospital Veterinário da UnB, por ter disponibilizado a estrutura necessária para a realização deste trabalho.

À Professora Christine, meu espelho profissional, minha eterna professora, que pacientemente foi me ensinando e abrindo minhas asas para voar dentro da Medicina Veterinária. Obrigada pelos conselhos que ajudaram a transformar as dificuldades em experiências profissionais.

À minha orientadora Profa Dra Gláucia Bueno, que demonstrou confiança na minha determinação para enfrentar os empecilhos que surgiriam na Graduação e no Mestrado e me supriu com conhecimento necessário para conseguir me impor de forma adequada perante as adversidades da profissão. Obrigada pela honra de ter a sua orientação durante e no final de cada etapa acadêmica.

Ao Dr. Rodrigo Cardoso Rabelo, pela orientação e apoio na condução deste trabalho. 


\section{SUMÁRIO}

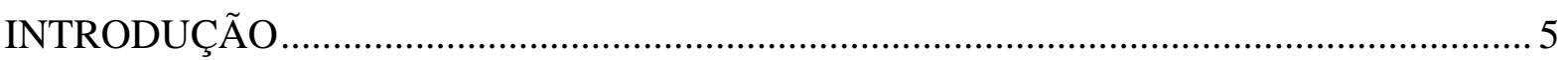

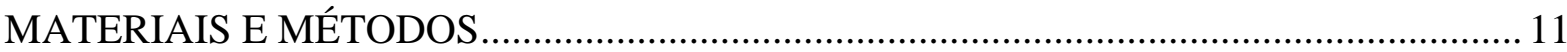

Seleção dos animais e avaliação dos parâmetros clínicos e laboratoriais .........................................11

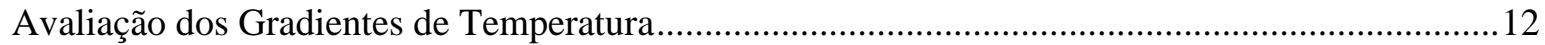

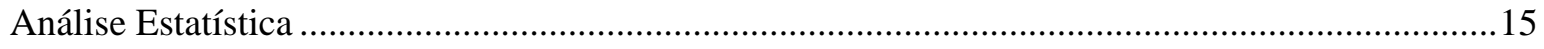

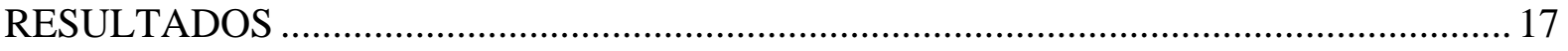

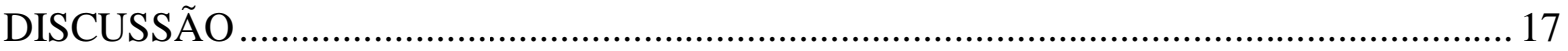

CONCLUSÃO

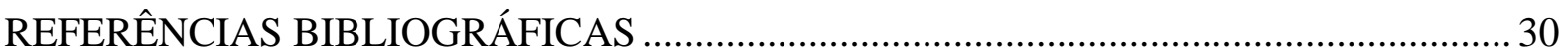




\section{LISTA DE ABREVIATURAS}

$\mathrm{Ta}$

$\Delta \mathrm{Tcp}$

$\Delta \mathrm{Tcp} 1$

$\Delta \mathrm{Tcp} 2$

TdMTE

TdMPE

TmMTE

$\Delta \mathrm{Tmd}$

$\Delta \mathrm{Tpa}$

$\Delta$ Tpa1

$\Delta \mathrm{Tpa} 2$

$\Delta \mathrm{Tsd}$
Temperatura ambiental

Gradiente de temperatura centro-periférica

Diferença entre temperatura retal e temperatura distal MTE

Diferença entre temperatura retal e temperatura distal MPE

Temperatura distal do membro torácico esquerdo

Temperatura distal do membro pélvico esquerdo

Temperatura medial do membro torácico esquerdo

Gradiente de temperatura member-diff: diferença entre temperatura distal do MTE

e temperatura distal do MPE

Gradiente de temperatura periférica-ambiente

Diferença entre temperatura distal do MTE e temperatura ambiental

Diferença entre temperatura distal do MPE e temperatura ambiental

Gradiente de temperatura skin-diff: diferença entre temperatura medial do MTE e temperatura distal do MTE. 


\section{LISTA DE FIGURAS}

Figuras 2. 1 (a): Aferição da temperatura do coxim palmar. 13

Figuras 2.1 (b): Aferição da temperatura da região proximal medial do rádio. 13

Figura 2.1 (c): Aferição da temperatura do coxim plantar. 13

Figura 2.2: Características da população sob estudo. 17 


\section{LISTA DE TABELAS}

Tabela 1. Análise descritiva das variáveis obtidas em gatos saudáveis.Erro! Indicador não definido.8

Tabela 2. Estudo do efeito de sexo sobre as variáveis estudadas 189

Tabela 3. Estudo do efeito de faixa etária sobre as variáveis

Tabela 4. Estudo do efeito da faixa de lactato sobre as temperaturas. 220

Tabela 5. Correlação entre as variáveis clínicas que indicam vasoconstrição periférica e os Gradientes de Temperatura em felinos. 


\section{Avaliação dos gradientes de temperatura em gatos hígidos}

K.S Morais ${ }^{\mathrm{a}}$, G. B. Pereira-Neto ${ }^{\mathrm{a}}$, R. C. Rabelo ${ }^{\mathrm{a}}, \mathrm{c}^{*}$, C.S. Martins ${ }^{\mathrm{a}}$, AR Lobo-Jr ${ }^{\mathrm{b}}$

a Hospital Veterinário da Faculdade de Agronomia e Medicina Veterinária, Universidade de

Brasília, Av. L4 Norte, Setor de Clubes Norte, Campus Universitário Darcy Ribeiro, Brasília, DF 70910-900, Brasil.

b Universidade Federal dos Vales do Jequitinhonha e Mucuri - Campus Unaí, Instituto de Ciências Agrárias, Unaí, MG, 38610-000, Brasil

c Intensivet Veterinary Consulting, Brasília, DF 70864-110, Brasil.

*Autor para correspondência. Tel.: 556183013196

Endereço de e-mail: intensivet@gmail.com (R.C. Rabelo) 


\section{RESUMO}

Objetivo - avaliar os diversos gradientes de temperatura em gatos hígidos e relacioná-los com a lactatemia, a glicemia e os parâmetros clínicos destes animais durante o exame físico de rotina.

Local: Hospital Veterinário de Universidade Pública

Métodos - foram triados 105 gatos, sendo que 98 animais foram incluídos para a coleta de dados que consistiu na mensuração das temperaturas periféricas (coxim palmar, plantar e região medial do rádio) e da central (pela via retal) para o cálculo dos gradientes (ou deltas) de temperatura. Os gradientes avaliados foram o centro-periférico, o periférico-ambiental, entre dois pontos do mesmo membro (skin-diff) e entre diferentes dois membros (limb-diff).

Resultados - os valores obtidos para as médias dos gradientes de temperatura em felinos saudáveis foram de $8^{\circ} \mathrm{C}$ para o centro-periférico; $7^{\circ} \mathrm{C}$ para o periférico-ambiental; $2,6^{\circ} \mathrm{C}$ para o skin-diff; e $0,1^{\circ} \mathrm{C}$ para limb-diff. Não ocorreu diferença estatística entre os parâmetros avaliados com relação aos membros utilizados (se torácicos ou pélvicos) ou em relação ao sexo. É possível que haja uma relação direta entre os gradientes de temperatura e a evolução da lactatemia e os sinais clínicos de vasoconstrição periférica.

Conclusões - os gradientes de temperatura são parâmetros que expressam a resposta fisiológica em felinos e podem ser avaliados de forma rápida e não invasiva, inclusive como meta hemodinâmica durante a reanimação de felinos graves. Neste estudo foi possível observar que felinos saudáveis podem apresentar sinais de vasoconstrição periférica e 
hiperlactatemia moderada durante a contenção mínima necessária para um exame físico de rotina. 


\begin{abstract}
Objective - to evaluate the various temperature gradients in healthy cats and to relate them to blood lactate, blood glucose and clinical parameters of these animals during routine physical examination.
\end{abstract}

Local: Veterinary Hospital of the Public University

Methods - 105 cats were screened, and 98 animals were included for data collection, which consisted of measurement of peripheral (palmar and plantar cushions and medial radius region) and central (measured rectally) temperatures to calculate the gradients (or deltas) of temperature. The evaluated gradients were the center-peripheral, peripheral-ambient, between two points of the same member (skin-diff) and between two members (limb-diff).

Results - The values for the mean temperature gradients in healthy cats were $8{ }^{\circ} \mathrm{C}$ for centralperipheral; $7^{\circ} \mathrm{C}$ for peripheral-ambient; $2.6^{\circ} \mathrm{C}$ for skin-diff and $0.1^{\circ} \mathrm{C}$ for limb-diff. There was no statistical difference between the evaluated parameters with respect to the members used (front or hind) or in relation to sex. There may be a direct relationship between the temperature gradients, the evolution of blood lactate concentration and clinical signs of peripheral vasoconstriction.

Conclusions - temperature gradients are parameters that express the physiological response in cats, and can be evaluated quickly and noninvasively, even as an hemodynamic goal during resuscitation of gravely ill felines. In this study, it was observed that healthy cats might show signs of peripheral vasoconstriction and moderate hyperlactatemia during the minimum containment required for a routine physical examination. 


\section{INTRODUÇÃO}

A condição fisiopatológica caracterizada pela incapacidade do sistema circulatório em fornecer quantidades de oxigênio e nutrientes para suprir as necessidades metabólicas dos tecidos é denominada "choque" (AKAMINE et al, 2010). Essa síndrome possui como mecanismo básico a perfusão tecidual inadequada que ocasiona a diminuição da oferta ou da utilização de oxigênio pelas células (WEIL e HENNING, 1979). As principais causas de base para a síndrome do choque são as alterações bruscas da volemia (e demais componentes da pré-carga), do tônus vascular (e demais componentes da pós-carga) ou do funcionamento da bomba cardíaca (AKAMINE et al, 2010). Com o objetivo de detectar o grau de comprometimento causado pela baixa perfusão e oxigenação tecidual, cada paciente deve ser submetido a sucessivas avaliações hemodinâmicas a fim de gerar uma intervenção precoce e evitar a disfunção orgânica (POLI DE FIGUEIREDO, SILVA e CORRÊA, 2008).

As principais respostas fisiológicas que o organismo mamífero utiliza para combater o estado de choque são a elevação do tônus simpático e a redução da atividade vagal eferente (RABELO e FERRARI, 2010). Para a preservação dos órgãos centrais, considerados vitais do ponto de vista circulatório (coração, pulmão e cérebro), esses mecanismos compensatórios serão ativados e promoverão a diminuição de fluxo sanguíneo e o aumento da resistência vascular periférica nos tecidos marginais, denominados periféricos (principalmente intestinos, rins, subcutâneo e pele). Vários estudos enfatizam que o leito vascular da circulação periférica é o primeiro a deteriorar-se e o último a ser restaurado após a reanimação do doente grave e por isso pode ser um bom indicador da perfusão (JOLY e WEIL, 1969; LIMA e BAKKER, 2005; LIMA et al, 2009; LIMA e BAKKER, 2014;). 
De forma geral, os felinos possuem uma resposta hemodinâmica diferenciada das demais espécies, no que diz respeito à velocidade de evolução do comprometimento circulatório. Os sistemas simpático e parassimpático dos felinos estão entrelaçados na região da base cardíaca, onde convergem todas as respostas agudas às diversas agressões hemodinâmicas. Sempre que houver um estímulo simpático excessivo, o sistema parassimpático promoverá uma resposta vagal que controle qualquer exagero. Desta forma, os sinais hiperdinâmicos que são comumente vistos na maioria dos mamíferos, ocorrerão de forma mais controlada nos felinos, reflexo conhecido como simpatovagal cardiocardíaco (SCHWARTZ et al, 1973). Todo esse mecanismo parece surgir de algumas particularidades da espécie, descritas no quadro 01.

Quadro 1. Particularidades hemodinâmicas na espécie felina

\begin{tabular}{|l|l|}
\hline \multicolumn{1}{|c|}{ Particularidade hemodinâmicas } & Referência \\
\hline Possuir uma frequência cardíaca basal mais alta, o que & \\
impede que qualquer queda brusca no débito cardíaco seja & \multirow{2}{*}{ SCHWARTZ et al, 1973 } \\
compensada inicialmente por taquicardia expressiva, como & \\
nas demais espécies mamíferas; & \\
relação a outros mamíferos, o que torna este sistema & CUNNINGHAM, 1993 \\
orgânico mais sensível aos eventos de vasoconstrição & \\
periférica e baixo fluxo; & \\
\hline
\end{tabular}




\begin{tabular}{|l|r|}
\hline $\begin{array}{l}\text { Possuir um menor volume sanguíneo por massa } \\
\text { corporal, cerca de 66mL/kg, aproximadamente } 25 \% \text { a }\end{array}$ & DAY, MACKIN e \\
menos que as demais espécies, e que torna o hipofluxo mais & LITTLEWOOD, 2000 \\
grave; & \\
\hline e a massa corporal total, aumentando a sensibilidade à & CUNNINGHAM, 1993 \\
hipotermia e ao baixo fluxo periférico. & \\
\hline
\end{tabular}

Dessa forma, os felinos demonstrarão uma descompensação circulatória sistêmica mais rápida quando sob estresse circulatório, evidenciada por alterações de vasoconstrição periférica mais precoce e menos duradoura, como: o pulso fraco ou não palpável; as membranas mucosas pálidas; o tempo de enchimento capilar superior a três segundos ou não evidente; a diminuição dos borborigmos intestinais e do débito urinário; além das alterações na linha hemodinâmica central, como frequência cardíaca normal ou diminuída; hipotensão arterial sistêmica; e uma profunda depressão mental precedidas pela hipotermia central sustentada (SCHWARTZ et al, 1973). Esta evolução pode culminar com a "hibernação termodependente" mais precoce nesta espécie, momento no qual o sistema simpático será desligado e o doente entrará em um “estado hipometabólico de descanso”, caracterizado por bradicardia, hipotermia grave, hipotensão arterial sistêmica, inconsciência e sinais de vasodilatação periférica, além de normo ou até mesmo hipolactatemia (RABELO, 2016).

Devido ao grau de comprometimento da perfusão periférica, causado pelo desvio de sangue para a linha central e pelo aumento da resistência vascular periférica, algumas modificações metabólicas serão iniciadas, como o desencadeamento da anaerobiose na linha periférica gerando o aumento do lactato (TOLEDO JÚNIOR e RABELO, 2012), e a 
diminuição do fluxo sanguíneo da pele gerando redução da temperatura cutânea (VINCENT, MORAINE e VAN DER LINDEN, 1988) (GENDEREN, LIMA e BOMMEL, 2012). Outro mecanismo fisiológico que pode gerar essas alterações é a liberação de adrenalina decorrente ao estresse da manipulação que acarreta a vasoconstrição periférica (OSTINI et al, 1998).

De forma geral, sabe-se que a avaliação do paciente crítico unicamente por meio dos parâmetros macrohemodinâmicos da linha central, como a pressão arterial sistêmica ou de oclusão da artéria pulmonar, o débito cardíaco, o nível de consciência e ou a temperatura central, pode ser insuficiente para a avaliação acurada da perfusão tecidual local e o diagnóstico precoce do estado de choque (RABELO e RIBEIRO, 2012), além de alguns destes métodos serem invasivos, podendo gerar complicações sistêmicas (GENDEREN, LIMA e BOMMEL, 2012).

Atualmente, existe uma tendência em utilizar os métodos de monitorização hemodinâmica menos invasivos e que possuam as características de serem confiáveis, realizados por qualquer operador, e financeiramente viáveis (GENDEREN, LIMA e BOMMEL, 2012). Os gradientes de temperatura possuem essas características e são considerados marcadores precoces de hipoperfusão tecidual, tornando-se importantes instrumentos de monitorização (JOLY e WEIL, 1969; LIMA et al, 2009).

A aferição simples da temperatura da pele é um parâmetro facilmente acessível utilizado para monitorizar a perfusão da periferia em pacientes críticos (JOLY e WEIL, 1969), porém as medições dos gradientes de temperatura podem gerar informações mais precisas sobre as alterações do fluxo sanguíneo cutâneo (RUBINSTEIN e SESSLER, 1990; AKATA et al, 2004). Os gradientes de temperatura corporal são determinados pela diferença de temperatura entre dois pontos de medição. Existem principalmente três tipos: o centro- 
periférico $(\Delta \mathrm{T} c \mathrm{p}), \quad$ o periférico-ambiental $(\Delta \mathrm{Tpa})$ e o do antebraço até a ponta do dedo ( $\triangle$ Tskin-diff) (GENDEREN, LIMA e BOMMEL, 2012).

Outro marcador de alteração na perfusão tecidual é a mensuração do lactato. A hiperlactatemia do tipo A, que ocorre devido a hipóxia tecidual secundária à hipoperfusão, à diminuição da oferta de oxigênio sanguíneo ou à dificuldade de mobilização de oxigênio pelos tecidos, é a mais comum. A hiperlactatemia do tipo B ocorre devido à alteração no metabolismo de carboidratos, pela diminuição da depuração do lactato ou mesmo durante a disfunção mitocondrial (ALLEN e HOLM, 2008). Por isso é sempre importante mensurar a lactatemia simultaneamente com a glicemia para construir um raciocínio clínico inicial sobre a origem do aumento do lactato.

Em particular, e em qualquer tipo de mecanismo de hiperlactatemia, a redução progressiva dos níveis de lactato ao longo do tempo reflete a restauração do fluxo sanguíneo tissular e o ajuste da entrega de oxigênio (HENNING, WEIL e WEINER, 1982; ANTONELLI et al, 2006).

Devido à importância da avaliação rápida e precoce da hipoperfusão periférica, e na ausência de estudos referentes ao comportamento da temperatura cutânea para a espécie Felis catus (gato doméstico), o objetivo primário deste estudo prospectivo observacional foi avaliar os gradientes de temperatura e sua associação com o valor do lactato, da glicemia e dos dados clínicos de gatos saudáveis durante a consulta clínica ambulatorial, a fim de auxiliar no diagnóstico de vasoconstrição periférica em situações de urgência.

Como hipótese, sugere-se que os felinos possuam um grau de estresse de contenção maior que o dos cães, ou dos humanos em ambiente hospitalar, e, por isso, podem apresentar maior vasoconstrição periférica durante o exame clínico de rotina. 
De forma secundária objetivamos comprovar se existe alguma diferença entre a mensuração da temperatura periférica no membro torácico ou no membro pélvico para gerar o cálculo dos gradientes de temperatura centro-periférico; se há diferença dos gradientes de temperatura entre gênero e na faixa etária de 1 a 7 anos e; se existe relação entre os diversos gradientes de temperatura com o lactato e a glicemia. 


\section{MATERIAIS E MÉTODOS}

O estudo foi aprovado pela Comissão de Ética no Uso Animal (CEUA) do Instituto de Ciências Biológicas da Universidade de Brasília, UnBDOC nº 90941/2014, em 19 de agosto de 2014. Todos os proprietários expressaram por escrito sua concordância em ceder seus animais.

Seleção dos animais e avaliação dos parâmetros clínicos e laboratoriais

Para a triagem dos animais deste estudo, realizou-se uma seleção prospectiva de gatos domésticos saudáveis, entre machos e fêmeas, de diferentes idades e raças. Os critérios de exclusão do estudo foram: possuírem alguma doença sistêmica crônica previamente diagnosticada; alguma dermatopatia ou lesão local que pudesse interferir na mensuração da temperatura da pele; e idade inferior a 1 ano ou superior a 7 anos. Apesar de não ter sido um dos fatores de exclusão, todos os animais do estudo eram castrados.

Os animais foram gentilmente posicionados sobre a mesa de atendimento coberta com toalhas para a realização do exame físico e, quando necessário, imobilizados apenas com a contenção manual da região dorsal do pescoço.

Nenhum dos animais demonstrou agressividade ou alto nível de estresse aparente durante o exame físico. A avaliação consistiu na aferição da frequência cardíaca, frequência respiratória, temperatura retal, coloração das membranas mucosas, tempo de preenchimento capilar, presença de borborigmos intestinais e nível de consciência, além de todas as temperaturas periféricas. 
A pressão arterial sistólica foi mensurada por meio de Doppler vascular (DV 610, Medmega $^{\circledR}$, Franca, Brasil) e esfigmomanômetro (Gamma G5, Heine ${ }^{\circledR}$, Herrsching, Alemanha), com braçadeira acoplada no membro torácico direito do animal em decúbito esternal, de acordo as diretrizes para a espécie (BROWN et al, 2007). O valor adotado foi a média aritmética de três medições consecutivas realizadas.

As amostras de sangue foram obtidas após o exame físico, por punção da veia femoral no membro pélvico direito. Após a coleta, foram realizadas imediatamente a aferição do lactato (Accutrend ${ }^{\circledR}$ Plus, Roche Diagnostics GmBH, Alemanha) e da glicemia (On Call ${ }^{\circledR}$ Plus, Roche Diagnostics GmBH, Alemanha) e logo após, o sangue foi colocado em tubos específicos para a realização de hemograma, determinação das concentrações séricas de creatinina, GamaGT, proteínas plasmáticas totais, albumina, globulina e alanina aminotransferase. O teste rápido para FIV e FeLV (imunoensaio cromatográfico - Alere ${ }^{\circledR}$ ) foi realizado posteriormente. Um único operador inspecionou todas as avaliações clínicas e exames complementares nos animais do estudo.

\section{Avaliação dos gradientes de temperatura}

Sequencialmente, após a aclimatação dos animais na temperatura ambiente do ambulatório durante 15 minutos, os gatos foram gentilmente posicionados em decúbito esternal e realizado o registro das seguintes temperaturas:

a) Ambiental (monitor multiparamétrico BIOPOC Bioeasy ${ }^{\circledR}$ ): A temperatura ambiente foi controlada por meio do equipamento de ar condicionado a uma temperatura de $24^{\circ} \mathrm{C}$, considerada termoneutra. 
b) Periférica do membro torácico esquerdo em dois pontos distintos: coxim palmar (Figura 2.1 a) e região proximal medial do rádio, sempre afastando os pelos (Figura 2.1b)

c) Periférica do membro pélvico esquerdo no coxim plantar (Figura 2.1c)

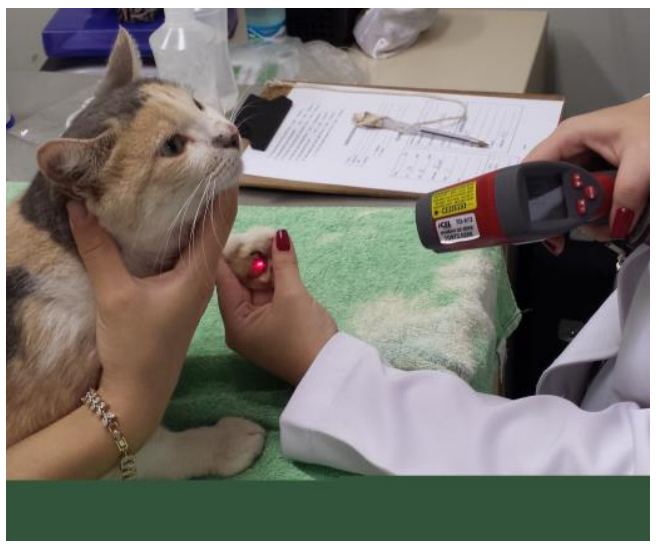

$2.1(\mathrm{a})$

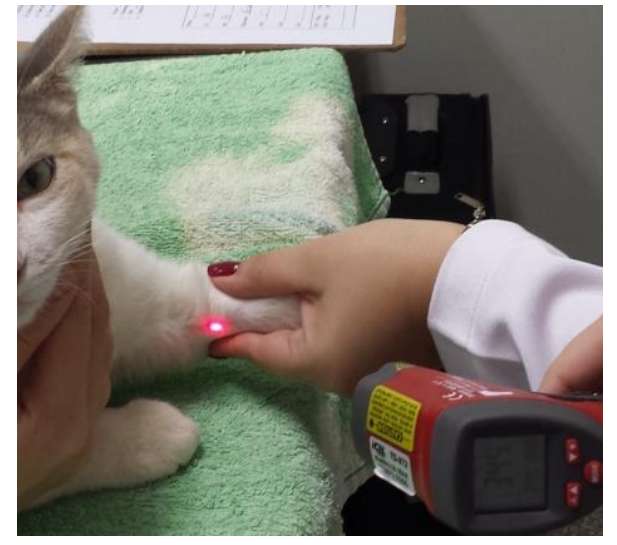

$2.1(\mathrm{~b})$

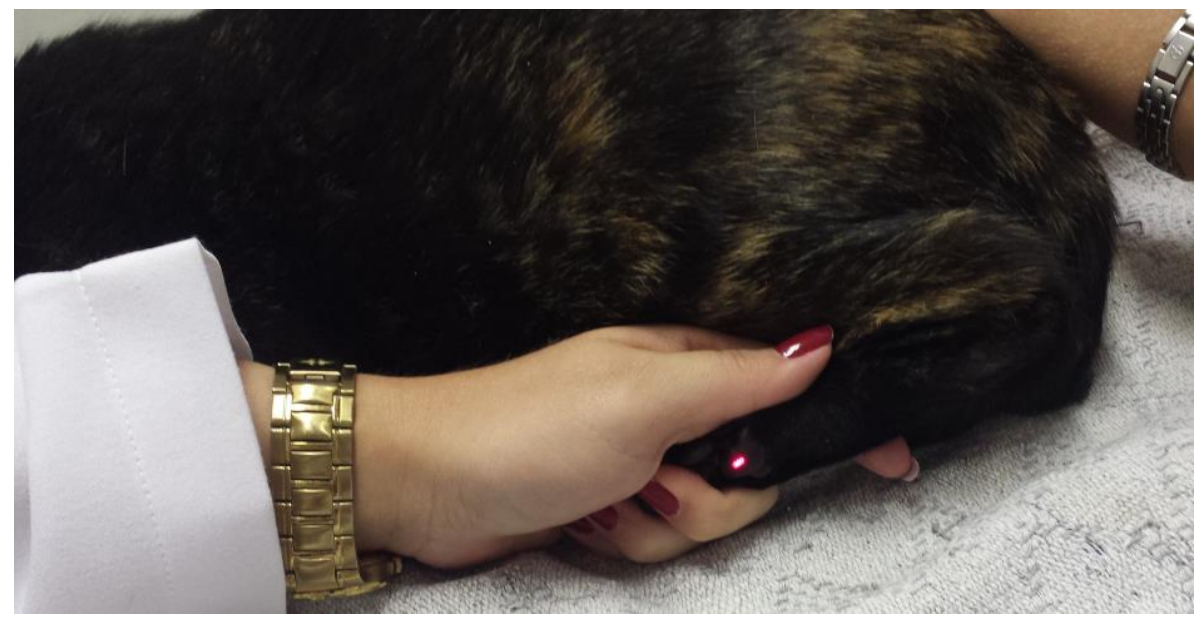

$2.1(\mathrm{c})$

Todas as temperaturas periféricas foram medidas por meio do termômetro infravermelho com duas ponteiras a laser (ST-700, Incotherm ${ }^{\circledR}$ ), emissividade ajustada em 
0.98, e em graus celsius $\left({ }^{\circ} \mathrm{C}\right.$ ) (VILLASEÑOR-MORA, SANCHES-MARIN e CALIXTOCORRERA, 2009). A distância do termômetro ao ponto de medição era de aproximadamente 13 centímetros (distância em que o laser das duas ponteiras se unem em um único ponto) e o laser foi deixado em contato com a pele durante 10 segundos para realizar cada medida. Os coxins foram escolhidos para o estudo por serem uma região distal glabra, de fluxo periférico terminal e de fácil acesso.

d) Central por via retal em ${ }^{\circ} \mathrm{C}$ (termômetro digital Rapid ${ }^{\circledR}$, Geratherm Medical do Brasil Ltda).

As temperaturas aferidas serviram de base para calcular os seguintes gradientes, expressos em ${ }^{\circ} \mathrm{C}$ :

a) Gradiente de temperatura centro-periférico $(\Delta \mathrm{Tcp})$ : diferença entre a temperatura central (retal) e a temperatura do coxim palmar do membro torácico esquerdo $(\Delta \mathrm{Tcp} 1)$ e a diferença entre a temperatura central (retal) e a temperatura do coxim plantar do membro pélvico esquerdo $(\Delta \mathrm{Tcp} 2)$;

b) Gradiente de temperatura em um mesmo membro ( $\Delta \mathrm{T}$ skin-diff ou $\Delta \mathrm{Tsd})$ : diferença de temperatura entre a região proximal do rádio no antebraço do membro torácico esquerdo e o coxim palmar no mesmo membro;

c) Gradiente de temperatura entre diferentes membros ( $\Delta$ Tlimb-diff ou $\Delta \mathrm{Tld})$ : baseado na diferença de temperatura entre o coxim palmar do membro torácico esquerdo e o coxim plantar do membro pélvico esquerdo;

d) Gradiente de temperatura periférico-ambiental ( $\Delta \mathrm{Tpa})$ : diferença entre as temperaturas ambiental e a do coxim palmar do membro torácico esquerdo ( $\Delta$ Tpa1); e 
diferença entre as temperaturas ambiental e do coxim plantar do membro pélvico esquerdo $(\Delta \mathrm{Tpa} 2)$

\section{Análise Estatística}

A análise foi realizada para descrever o comportamento dos dados. Em seguida, uma análise de variância (ANOVA) foi realizada para as variáveis segundo um delineamento inteiramente casualizado (DIC) em um arranjo fatorial 3 (grupo: grupo 1, grupo 2 e grupo 3 ) $\times$ 2 (sexo: macho e fêmea) $\times 2$ (faixa etária: $\leq 36$ e $>36$ meses), usando um total de 98 gatos (37 machos e 61 fêmeas). Neste caso, os efeitos fixos de grupo, sexo, faixa etária e suas interações foram considerados no modelo. Além disso, o peso dos animais foi usado como covariável no modelo. Por fim, uma análise de correlação com os dados brutos e com os resíduos foi conduzida para verificar associações entre algumas variáveis.

A análise descritiva, ANOVA e correlação dos dados foram conduzidas usando respectivamente os procedimentos MEANS, MIXED e CORR do software Statistical Analysis Sytem (SAS; versão 9.2). Para considerar um efeito significante na ANOVA, um nível de probabilidade de $1 \%$ ou menor para o teste $F$ foi adotado. As médias de quadrado mínimo e os erros padrões foram apresentados para todas variáveis nas ANOVAs. O teste $t$ de Student foi aplicado para discriminar as médias quando efeitos significantes foram observados para fatores com mais do que dois níveis.

Depois dos dados iniciais, uma segunda abordagem com ANOVA foi realizada para as variáveis segundo um DIC, para estudar o efeito de diferentes faixas de lactato $(<3,3$ - 5 e $>5 \mathrm{mmol} / \mathrm{L}$ ). Para isso, somente o efeito fixo de faixa de lactato foi considerado no modelo. Além disso, ANOVAs também foram conduzidas para estudar o efeito de diferentes 
faixas de $\Delta \mathrm{TCP} 1\left(<7,7-10\right.$ e $\left.>10{ }^{\circ} \mathrm{C}\right)$ e $\Delta \mathrm{TCP} 2\left(<7,7-10\right.$ e $\left.>10{ }^{\circ} \mathrm{C}\right)$ sobre as variáveis lactato e vascularização. Desta forma, um modelo contemplava o efeito fixo de faixa de $\Delta \mathrm{Tcp} 1$ e um outro modelo contemplava o efeito fixo de $\Delta \mathrm{Tcp} 2$ para as duas variáveis.

As ANOVAs dos dados foram conduzidas usando o procedimento MIXED do software SAS. Os critérios adotados para considerar um efeito significante e o teste de médias aplicado nos dados foram os mesmos já descritos. 


\section{RESULTADOS}

Todas as variáveis apresentaram como resultado uma distribuição estatística normal. Após a análise dos resultados obtidos em 105 gatos, 7 animais foram excluídos do estudo por possuírem FiV ou FelV. A Figura 2 demonstra as características gerais da população felina sob estudo

Figura 2.2: Características da população sob estudo.

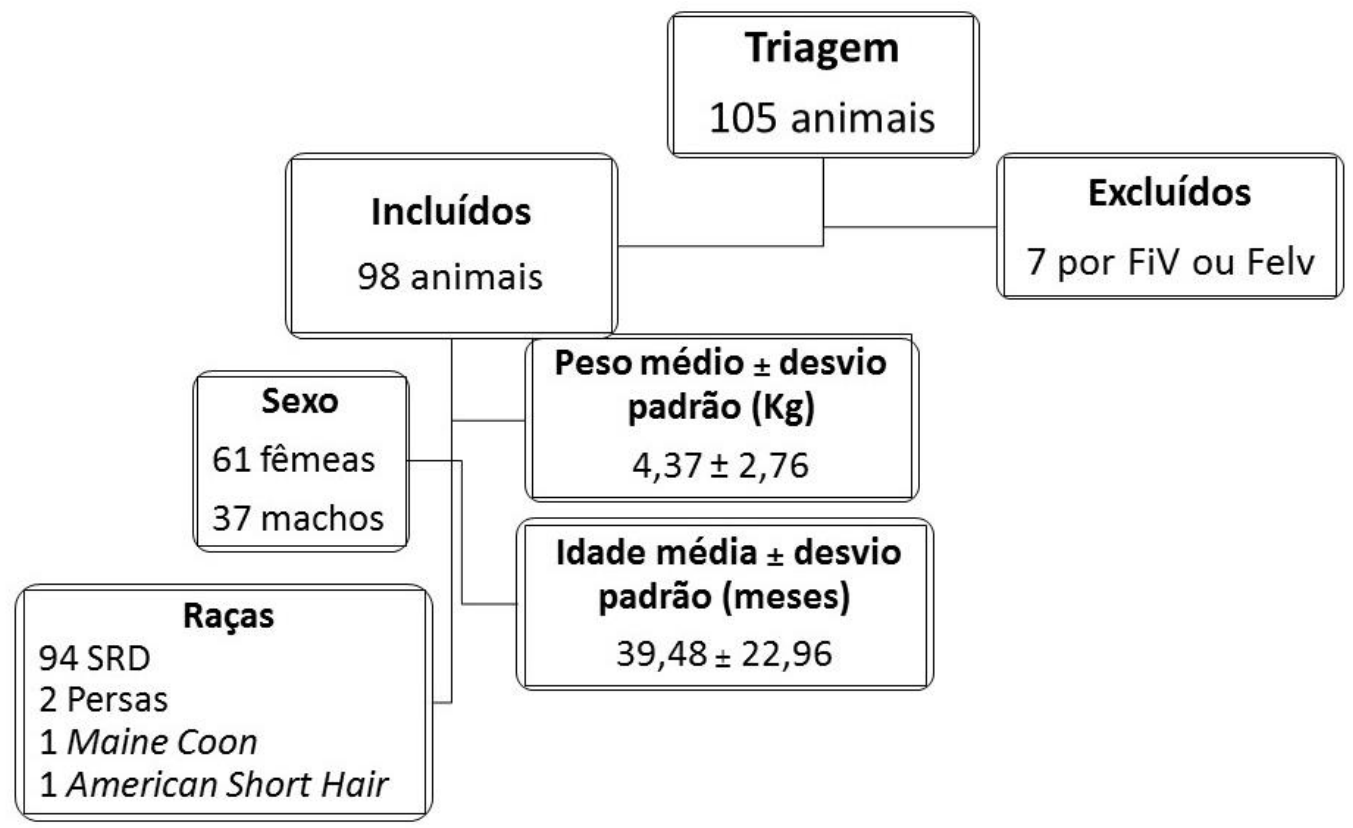

A tabela 1 descreve os valores obtidos de 98 animais com relação à glicemia e lactato sanguíneos, além das temperaturas medidas em diversos pontos. 
Tabela 1. Análise descritiva das variáveis clínicas e laboratoriais obtidas em felinos.

\begin{tabular}{llllll}
\hline Variável & $\mathrm{N}$ & Média & Desvio Padrão & Mínimo & Máximo \\
\hline Glicemia & 98 & 84,3 & 16,27 & 60,0 & 143,0 \\
Lactato $(\mathrm{mmol} / \mathrm{L})$ & 98 & 3,4 & 0,87 & 1,4 & 6,8 \\
Temperatura retal $\left({ }^{\circ} \mathrm{C}\right)$ & 98 & 38,5 & 0,57 & 37,2 & 39,9 \\
Td MTE $\left({ }^{\circ} \mathrm{C}\right)$ & 98 & 30,3 & 1,84 & 25,2 & 34,2 \\
Tm MTE $\left({ }^{\circ} \mathrm{C}\right)$ & 98 & 32,9 & 1,52 & 29,2 & 36,8 \\
Td MPE $\left({ }^{\circ} \mathrm{C}\right)$ & 98 & 30,2 & 2,13 & 25,5 & 34,5 \\
Ta $\left({ }^{\circ} \mathrm{C}\right)$ & 98 & 23,0 & 1,54 & 19,6 & 27,2 \\
$\Delta \operatorname{Tcp} 1\left({ }^{\circ} \mathrm{C}\right)$ & 98 & 8,2 & 1,89 & 3,5 & 13,2 \\
$\Delta \operatorname{Tcp} 2\left({ }^{\circ} \mathrm{C}\right)$ & 98 & 8,4 & 2,08 & 4,2 & 13,1 \\
$\Delta \operatorname{Tpa} 1\left({ }^{\circ} \mathrm{C}\right)$ & 98 & 7,3 & 1,98 & 1,1 & 11,5 \\
$\Delta \operatorname{Tp} 2\left({ }^{\circ} \mathrm{C}\right)$ & 98 & 7,2 & 2,25 & 0,7 & 14,7 \\
$\Delta \operatorname{Tsd}\left({ }^{\circ} \mathrm{C}\right)$ & 98 & 2,6 & 1,34 & 0,0 & 6,4 \\
$\Delta \operatorname{Tlimb}-\operatorname{diff}\left({ }^{\circ} \mathrm{C}\right)$ & 98 & 0,1 & 1,42 & $-4,2$ & 3,6 \\
$\operatorname{PAS} \mathrm{mmHg}$ & 98 & 131,2 & 14,87 & 109,3 & 160
\end{tabular}

Legendas: Td MTE (Temperatura distal do membro torácico esquerdo); Tm MTE (Temperatura medial do membro torácico esquerdo); Td MPE (Temperatura distal do membro pélvico esquerdo); Ta (Temperatura ambiental); $\Delta \mathrm{Tcp} 1$ (Gradiente de temperatura Centro Periférico 1); $\Delta \mathrm{Tcp} 2$ (Gradiente de temperatura Centro Periférico 2); $\Delta$ Tpa1 (Gradiente de temperatura Periférico Ambiental 1); $\Delta \mathrm{Tpa} 2$ (Gradiente de temperatura Periférico Ambiental 2); $\Delta$ Tsd (Gradiente de temperatura Skin Diff 1); $\Delta$ Tlimb-diff (Gradiente de temperatura Limb-Diff); PAS (Pressão Arterial Sistólica).

Para o estudo do efeito do sexo sobre as variáveis medidas, os animais foram agrupados em machos e fêmeas. Neste caso não ocorreu diferença estatisticamente significativa entre os grupos para a glicemia e lactato sanguíneos, além das temperaturas medidas em diversos pontos, conforme demonstrado na tabela 2 . 
Tabela 2. Estudo do efeito do sexo sobre as variáveis estudadas.

\begin{tabular}{|c|c|c|c|}
\hline \multirow{2}{*}{ Variável } & \multicolumn{2}{|l|}{ Sexo } & \multirow{2}{*}{-Valor de $P$} \\
\hline & Macho (n=37) & Fêmea $(\mathrm{n}=61)$ & \\
\hline Glicemia & $84,3(2,94)$ & $81,3(2,37)$ & 0,45 \\
\hline Lactato mmol / L & $3,3(0,18)$ & $3,4 \pm 0,15$ & 0,67 \\
\hline Temperatura retal $\left({ }^{\circ} \mathrm{C}\right)$ & $38,4(0,11)$ & $38,6 \pm 0,09$ & 0,22 \\
\hline $\operatorname{Td} \operatorname{MTE}\left({ }^{\circ} \mathrm{C}\right)$ & $30,4(0,36)$ & $30,2 \pm 0,29$ & 0,69 \\
\hline $\operatorname{Tm}$ MTE $\left({ }^{\circ} \mathrm{C}\right)$ & $33,0 \quad(0,30)$ & $32,8 \pm 0,24$ & 0,50 \\
\hline $\operatorname{Td} \operatorname{MPE}\left({ }^{\circ} \mathrm{C}\right)$ & $29,8 \pm 0,39$ & $30,2 \pm 0,31$ & 0,38 \\
\hline $\mathrm{Ta}\left({ }^{\circ} \mathrm{C}\right)$ & $22,8 \pm 0,32$ & $23,1 \pm 0,25$ & 0,48 \\
\hline$\Delta \mathrm{Tcp} 1\left({ }^{\circ} \mathrm{C}\right)$ & $8,0 \pm 0,37$ & $8,4 \pm 0,30$ & 0,45 \\
\hline$\Delta \mathrm{Tcp} 2\left({ }^{\circ} \mathrm{C}\right)$ & $8,7 \pm 0,39$ & $8,4 \pm 0,31$ & 0,61 \\
\hline$\Delta \mathrm{Tpa} 1\left({ }^{\circ} \mathrm{C}\right)$ & $7,6 \pm 0,39$ & $7,1 \pm 0,31$ & 0,35 \\
\hline$\Delta \mathrm{Tpa} 2\left({ }^{\circ} \mathrm{C}\right)$ & $7,0 \pm 0,43$ & $7,1 \pm 0,35$ & 0,79 \\
\hline$\Delta \operatorname{Tsd}\left({ }^{\circ} \mathrm{C}\right)$ & $2,6 \pm 0,27$ & $2,6 \pm 0,22$ & 0,82 \\
\hline$\Delta \mathrm{T} \operatorname{limb}-\operatorname{diff}\left({ }^{\circ} \mathrm{C}\right)$ & $0,64 \pm 0,27$ & $-0,01 \pm 0,22$ & 0,08 \\
\hline PAS mmHg & $128,6 \pm 2,98$ & $131,9 \pm 2,38$ & 0,41 \\
\hline
\end{tabular}

Média de quadrado mínimo (erro padrão).

Legendas: Td MTE (Temperatura distal do membro torácico esquerdo); Tm MTE (Temperatura medial do membro torácico esquerdo); Td MPE (Temperatura distal do membro pélvico esquerdo); Ta (Temperatura ambiental); $\Delta \mathrm{Tcp} 1$ (Diferença entre temperatura retal e Temp. distal MTE); $\Delta \mathrm{Tcp} 2$ (Diferença entre temperatura retal e Temp. distal MPE); $\Delta \mathrm{Tpa}$ (Diferença entre temperatura distal do MTE e temperatura ambiental); $\Delta \mathrm{Tpa} 2$ (Diferença entre temperatura distal do MPE e temperatura ambiental); $\Delta$ Tsd (Diferença entre temperatura medial do MTE e temperatura distal do MTE); $\Delta$ Tlimb-diff (Diferença entre temperatura distal do MTE e temperatura distal do MPE); PAS (Pressão Arterial Sistólica).

Para o estudo do efeito da faixa etária sobre as variáveis, os animais foram alocados em dois grupos, sendo o Grupo A composto por animais com idade entre 12 meses e até 36 meses; e Grupo B por animais com idade superior aos 36 meses e até 84 meses (tabela $3)$. 
Tabela 3. Estudo do efeito da faixa etária sobre as variáveis estudadas.

\begin{tabular}{|c|c|c|c|}
\hline \multirow{2}{*}{ Variável } & \multicolumn{2}{|l|}{ Grupos } & \multirow{2}{*}{-Valor de $P$} \\
\hline & Grupo A $(\mathrm{n}=57)$ & Grupo B $(n=41)$ & \\
\hline Glicemia & $79,6 \pm 2,16$ & $85,9 \pm 2,85$ & 0,08 \\
\hline Lactato (mmol / L) & $3,3 \pm 0,13$ & $3,4 \pm 0,17$ & 0,48 \\
\hline Temperatura retal $\left({ }^{\circ} \mathrm{C}\right)$ & $38,7 \pm 0,08$ & $38,4 \pm 0,11$ & 0,03 \\
\hline $\operatorname{Td} \operatorname{MTE}\left({ }^{\circ} \mathrm{C}\right)$ & $30,6 \pm 0,26$ & $30,0 \pm 0,35$ & 0,16 \\
\hline Tm MTE $\left({ }^{\circ} \mathrm{C}\right)$ & $33,0 \pm 0,22$ & $32,7 \pm 0,29$ & 0,40 \\
\hline $\mathrm{Td} \operatorname{MPE}\left({ }^{\circ} \mathrm{C}\right)$ & $30,6 \pm 0,29^{a}$ & $29,4 \pm 0,37^{\mathrm{b}}$ & 0,01 \\
\hline $\mathrm{Ta}\left({ }^{\circ} \mathrm{C}\right)$ & $23,2 \pm 0,23$ & $22,7 \pm 0,31$ & 0,16 \\
\hline$\Delta \mathrm{Tcp} 1\left({ }^{\circ} \mathrm{C}\right)$ & $8,1 \pm 0,27$ & $8,4 \pm 0,36$ & 0,49 \\
\hline$\Delta \mathrm{Tcp} 2\left({ }^{\circ} \mathrm{C}\right)$ & $8,1 \pm 0,29$ & $9,0 \pm 0,37$ & 0,06 \\
\hline$\Delta \mathrm{Tpa} 1\left({ }^{\circ} \mathrm{C}\right)$ & $7,4 \pm 0,29$ & $7,3 \pm 0,38$ & 0,87 \\
\hline$\Delta \mathrm{Tpa} 2\left({ }^{\circ} \mathrm{C}\right)$ & $7,4 \pm 0,32$ & $7,0 \pm 0,43$ & 0,20 \\
\hline$\Delta \operatorname{Tsd}\left({ }^{\circ} \mathrm{C}\right)$ & $2,4 \pm 0,20$ & $2,7 \pm 0,26$ & 0,35 \\
\hline$\Delta \mathrm{T}$ limb-diff $\left({ }^{\circ} \mathrm{C}\right)$ & $0,01 \pm 0,20$ & $0,61 \pm 0,26$ & 0,08 \\
\hline PAS mmHg & $128,2 \pm 2,19$ & $132,3 \pm 2,86$ & 0,25 \\
\hline
\end{tabular}

Média de quadrado mínimo (erro padrão). ${ }^{\text {a,b } M e ́ d i a s ~ s e g u i d a s ~ p o r ~ l e t r a s ~ m i n u ́ s c u l a s ~ d i f e r e n t e s ~ e n t r e ~ a s ~ f a i x a s ~}$ etárias diferem estatisticamente pelo teste $F$ a um nível de probabilidade de $1 \%$.

Legendas: Td MTE (Temperatura distal do membro torácico esquerdo); Tm MTE (Temperatura medial do membro torácico esquerdo); Td MPE (Temperatura distal do membro pélvico esquerdo); Ta (Temperatura ambiental); $\Delta \mathrm{Tcp} 1$ (Gradiente de temperatura Centro Periférico 1); $\Delta \mathrm{Tcp} 2$ (Gradiente de temperatura Centro Periférico 2); $\Delta$ Tpa1 (Gradiente de temperatura Periférico Ambiental 1); $\Delta \mathrm{Tpa} 2$ (Gradiente de temperatura Periférico Ambiental 2); $\Delta$ Tsd (Gradiente de temperatura Skin Diff 1); $\Delta$ Tlimb-diff (Gradiente de temperatura limb-diff); PAS (Pressão Arterial Sistólica).

Houve diferença estatística apenas na medida da temperatura distal do MPE, quando o Grupo A apresentou a temperatura do MPE mais alta em relação ao Grupo B. Em relação ao lactato, não houve diferença estatisticamente significativa em nenhuma das análises supracitadas. 
Em seguida, o $\mathrm{N}$ total de 98 animais foi dividido em 3 grupos afim de testar as variáveis de temperatura em relação aos diferentes níveis de lactato sanguíneo, e também nesse caso não ocorreu diferença estatisticamente significativa entre os grupos analisados, como demonstrado na tabela 4.

Os níveis de lactato sanguíneo foram divididos da seguinte maneira:

1. Grupo L1 possuindo o valor de Lactato menor que $3 \mathrm{mmol} / \mathrm{L}$;

2. Grupo L2 de 3 a $5 \mathrm{mmol} / \mathrm{L}$;

3. Grupo L3 maior que $5 \mathrm{mmol} / \mathrm{L}$

Tabela 4. Estudo do efeito da faixa de lactato sobre as temperaturas periféricas em felinos.

\begin{tabular}{lcccc}
\hline Variável $\left({ }^{\circ} \mathrm{C}\right)$ & \multicolumn{3}{c}{ Grupos } & Valor de $P$ \\
\cline { 2 - 4 } & $\mathrm{L} 1(\mathrm{n}=26)$ & $\mathrm{L} 2(\mathrm{n}=67)$ & $\mathrm{L} 3(\mathrm{n}=5)$ & 0,71 \\
\hline Temp. distal MTE & $30,5 \pm 0,36$ & $30,3 \pm 0,23$ & $29,9 \pm 0,83$ & 0,43 \\
Temp.medial MTE & $32,9 \pm 0,30$ & $32,8 \pm 0,19$ & $33,7 \pm 0,68$ & 0,58 \\
Temp. distal MPE & $30,5 \pm 0,42$ & $30,2 \pm 0,26$ & $29,4 \pm 0,96$ & 0,36 \\
Temp. amb. & $22,7 \pm 0,30$ & $23,1 \pm 0,19$ & $23,7 \pm 0,69$ & 0,72 \\
$\Delta$ TCP1 & $8,0 \pm 0,37$ & $8,3 \pm 0,23$ & $8,7 \pm 0,85$ & 0,57 \\
$\Delta$ TCP2 & $8,1 \pm 0,41$ & $8,4 \pm 0,26$ & $9,1 \pm 0,94$ & 0,17 \\
$\Delta$ TPA1 & $7,8 \pm 0,39$ & $7,2 \pm 0,24$ & $6,1 \pm 0,88$ & 0,15 \\
$\Delta$ TPA2 & $7,7 \pm 0,44$ & $7,1 \pm 0,27$ & $5,7 \pm 1,00$ & 0,07 \\
$\Delta$ Tlimb-diff & $2,4 \pm 0,26$ & $2,5 \pm 0,16$ & $3,9 \pm 0,59$ & 0,87 \\
$\Delta$ TSD & $0,08 \pm 0,28$ & $0,14 \pm 0,17$ & $0,45 \pm 0,64$ &
\end{tabular}

Média de quadrado mínimo (erro padrão).

Legendas: Td MTE (Temperatura distal do membro torácico esquerdo); Tm MTE (Temperatura medial do membro torácico esquerdo); Td MPE (Temperatura distal do membro pélvico esquerdo); Ta (Temperatura ambiental); $\Delta$ Tcp1 (Gradiente de temperatura Centro Periférico 1); $\Delta \mathrm{Tcp} 2$ (Gradiente de temperatura Centro Periférico 2); $\Delta$ Tpa1 (Gradiente de temperatura Periférico Ambiental 1); $\Delta \mathrm{Tpa} 2$ (Gradiente de temperatura Periférico Ambiental 2); $\Delta$ Tlimb-diff (Gradiente de temperatura limb-diff); $\Delta$ Tsd (Gradiente de temperatura skindiff ). 
Uma nova tabela (Tabela 5) foi realizada para correlacionar marcadores clínicos de hipoperfusão periférica com os gradientes de temperatura. Para a análise desses marcadores, foi dada uma pontuação de 0 para a variável normal e 1 para indicar vasoconstrição, a citar:

Borborigmos Intestinais (medidos em 3 minutos de ausculta abdminal):

1. Pontuação 0: borborigmos auscultados

2. Pontuação 1: ausência de borborigmos auscultados

TPC (tempo de preenchimento capilar):

3. Pontuação 0: TPC igual a 2 segundos

4. Pontuação 1: TPC menor que 1 segundo

Tabela 5: Correlação entre as variáveis clínicas que indicam vasoconstrição periférica e os Gradientes de Temperatura em felinos.

\begin{tabular}{ccccc} 
Variável & $\Delta$ Tcp1 & $\Delta$ Tcp2 & $\Delta$ Tld & $\Delta$ Tsd \\
\hline TPC & $0,57^{*}\left(0,58^{*}\right)$ & $0,61 *\left(0,65^{*}\right)$ & $0,19^{*}\left(0,19^{*}\right)$ & $0,14(0,14)$ \\
Borborigmos & $-0,13(-0,16)$ & $0,03(-0,05)$ & $-0,01(-0,07)$ & $0,21 *(0,15)$
\end{tabular}

Média de quadrado mínimo (erro padrão). *Valores estatisticamente significativos.

Legendas: TPC (Tempo de Preenchimento Capilar); $\triangle \mathrm{Tcp} 1$ (Gradiente de temperatura Centro Periférico 1); $\Delta$ Tcp2 (Gradiente de temperatura Centro Periférico 2); $\Delta$ Tld (Gradiente de temperatura limb-diff 1); $\Delta$ Tsd (Gradiente de temperatura skin-diff). 


\section{DISCUSSÃO}

Neste estudo prospectivo observacional incluindo 98 gatos saudáveis, demonstrou-se para a população avaliada que os gradientes de temperatura e o lactato são análises independentes e que podem ser utilizados em machos e fêmeas de 1 a 7 anos de idade para gerar parâmetros que auxiliem o clínico na rotina médica de felinos.

$\mathrm{O}$ valor médio do gradiente de temperatura centro-periférica $(\Delta \mathrm{Tcp})$ obtido para gatos clinicamente saudáveis foi de aproximadamente $8^{\circ} \mathrm{C}$, sendo mais alto do que os valores reportados em humanos ou cães. Este fato por ser decorrente do comportamento dos gatos em ambientes estressantes, com maior excitação, levando-os a gerar maior liberação de adrenalina que ocasiona vasoconstrição reflexa das arteríolas da pele (OSTINI et al, 1998; KOCIBA, 2004).

Os resultados também sugerem que em gatos clinicamente saudáveis não há diferença estatística significativa entre o $\Delta \mathrm{Tcp} 1$ e o $\Delta \mathrm{Tcp} 2$ como demonstrado pelos valores médios obtidos $\left(8,2 \pm 1,89^{\circ} \mathrm{C}\right.$ e $8,4 \pm 2,08^{\circ} \mathrm{C}$, respectivamente), ou seja, para mensurar o $\Delta \mathrm{Tcp}$ em gatos, a aferição poderia ser realizada tanto no membro torácico, quanto no pélvico. De qualquer forma, sugere-se que a medida seja realizada como padrão no membro pélvico esquerdo, assim como em cães, já que a manipulação destes membros durante a internação tende a ser mais reduzida, pois os acessos vasculares são preferencialmente colocados nos membros torácicos.

O $\Delta$ Tcp é uma variável que auxilia na análise indireta da hipoperfusão tecidual decorrente da vasoconstrição periférica. Nos estados de choque, essa vasoconstrição ocasiona a diminuição da temperatura da pele para manter a macrocirculação central estável, resultando 
no aumento da diferença da temperatura centro-periférica (ALDRICH, 2005). O valor em humanos saudáveis situa-se entre 3 e $7^{\circ} \mathrm{C}$ (CURLEY e SMYRNIOS, 1990; GENDEREN, LIMA e BOMMEL, 2012). O valor médio normal para espécie canina é de 6 a $7^{\circ} \mathrm{C}$, sendo que os valores maiores que $10^{\circ} \mathrm{C}$ são considerados altamente prejudiciais (LIMA e BAKKER, 2005; RABELO e RIBEIRO, 2012).

Os gradientes de temperatura periférico-ambiental ( $\Delta \mathrm{Tpa})$ neste estudo não demonstraram diferença estatística significativa quando comparados entre si e obtiveram os valores médios de $7,3 \pm 1,98^{\circ} \mathrm{C}$ para $\Delta \mathrm{Tpa} 1$ e de $7,2 \pm 2,25^{\circ} \mathrm{C}$ para $\Delta \mathrm{Tpa}$. Portanto, pode-se inferir a partir destes resultados, que o valor de $\Delta$ Tpa para a população deste estudo é de aproximadamente $7^{\circ} \mathrm{C}$ em qualquer um dos membros esquerdos (torácico ou pélvico) os quais podem ser utilizados para a aferição deste gradiente.

$\mathrm{O}$ gradiente de temperatura periférico-ambiental $(\Delta \mathrm{Tpa})$ indica que variações na temperatura da pele quando o organismo é mantido em uma temperatura ambiente termoneutra (entre 21 a $24^{\circ} \mathrm{C}$ ) sugerem alterações do fluxo sanguíneo local (LIMA e BAKKER, 2005)(VIVEIROS, MEYER e KRUEL, 2009). O valor da temperatura ambiente neste trabalho foi controlado e apresentou média de $23 \pm 1,54^{\circ} \mathrm{C}$. Relatos em humanos descrevem que valores de $\Delta$ Tpa próximos a $7^{\circ}$ são compatíveis com a normalidade e que valores menores que $4^{\circ} \mathrm{C}$ aumentam a probabilidade de óbito em pacientes em choque (JOLY e WEIL, 1969). Neste estudo, sugerimos que os valores médios de normalidade de $\Delta$ Tpa para humanos e gatos domésticos são similares, no entanto, mais estudos serão necessários para inferir valores preditivos de mortalidade em felinos enfermos.

Em relação ao gradiente de temperatura do antebraço até a ponta do dedo, ou "skin-diff " $(\Delta \mathrm{Tsd})$, o presente estudo encontrou o valor médio de 2,6 $\pm 1,34^{\circ} \mathrm{C}$. Estudos em humanos relataram valores de $\Delta \mathrm{Tsd}$ próximos a $0^{\circ} \mathrm{C}$ quando não há vasoconstrição, e maiores 
que $4^{\circ} \mathrm{C}$ durante a vasoconstrição grave (HOUSE e TIPTON, 2002; GENDEREN, LIMA e BOMMEL, 2012). Para a espécie canina, o valor recomendado é de $1,3^{\circ} \mathrm{C}$ (RABELO e FERRARI, 2010), ou seja, o presente estudo sugere que o $\Delta \mathrm{Tsd}$ em gatos é $1,3^{\circ} \mathrm{C}$ maior do que o relatado para cães. Infere-se que essa diferença ocorreu devido a uma vasoconstrição mais acentuada na parte distal do membro em relação a parte proximal no antebraço, provavelmente pelo estresse resultante da contenção nesta espécie.

O $\Delta$ Tsd é utilizado para identificar o início da vasoconstrição em pacientes no trans e no pós operatório, quando o reflexo simpático pode estar comprometido. Durante a cirurgia, a hipotermia ocorre devido a alguns fatores, entre eles as baixas temperaturas do centro cirúrgico, a exposição corporal e de superfícies viscerais ao ambiente e a supressão da resposta fisiológica pela anestesia (como a ausência de tremores). Esse gradiente é obtido pela a diferença entre a temperatura de dois pontos no mesmo membro. Para tal aferição, esse membro deve ser posicionado sem inclinação e exposto à mesma temperatura ambiente, desta forma, pode-se sugerir que a diferença de temperatura entre o antebraço e a ponta do dedo é decorrente da vasoconstrição periférica (HOUSE e TIPTON, 2002; GENDEREN, LIMA e BOMMEL, 2012).

O gradiente de temperatura entre membros ( $\Delta$ Tlimb-diff), sugerido como uma nova medida neste trabalho, apresentou o valor médio de $0,1 \pm 1,42{ }^{\circ} \mathrm{C}$. Subentende-se com este resultado que existe uma variação mínima de temperatura entre os membros hemilateriais, portanto valores maiores que o referido poderia sugerir uma alteração da homogeneidade de perfusão. Em humanos, os pacientes são considerados portadores de extremidades frias se algum dos pontos distais de aferição (dedos das mãos e pés) estiverem em uma temperatura abaixo do normal, quando na ausência de doença vascular periférica oclusiva (GENDEREN, LIMA e BOMMEL, 2012). 
Em relação ao gênero dos animais avaliados, não ocorreu diferença estatística entre machos e fêmeas e os valores médios de todos os gradientes estudados podem ser utilizados para ambos os sexos. Por sua vez, em relação à faixa etária, ocorreu diferença estatística apenas na Temperatura distal do MPE, com o Grupo A demonstrando maiores valores em relação ao Grupo B. Esse resultado difere do relato em que a resposta da amplitude vasoconstritora varia de acordo com a idade do paciente, diminuindo em pacientes mais velhos (FEGEREMAIL e BRAUNE, 2005). É provável que a parcela de animais com maior idade neste estudo fosse mais sensível ao estresse de contenção que o outro grupo, no entanto, esta alteração não parece significante do ponto de vista do gradiente centroperiférico.

Apesar de todos os benefícios citados sobre a aferição dos gradientes de temperatura, o método deve ser usado com cautela, uma vez que pode ser mal interpretado e não refletir vasoconstrição periférica em situações como a doença arterial periférica, hipotermia central grave com hibernação termodependente, choque com vasodilatação e administração de fármacos de ação vasoconstritoras (RÉA-NETO et al, 2006; RABELO, 2016).

Em relação ao lactato, existem estudos para tentar estabelecer o seu valor para cães, sendo o referencial em adultos saudáveis de 0,2 a 2,5 mmol/L (TOLEDO JÚNIOR e RABELO, 2012). Em gatos, sua utilidade clínica tem sido questionada, devido ao estresse que o animal passa no momento da punção venosa que pode gerar hiperlactatemia mesmo em animais saudáveis (RAND et al, 2002; REDAVID et al, 2012). Apesar disso, um estudo recente com gatos saudáveis encontrou o valor de 0,39 a 2,87 $\mathrm{mmol}$ / L, porém os gatos foram mantidos internados para simular a variação de lactato que poderia existir durante o estresse da internação (REDAVID et al, 2012). Em outro estudo, Rand et al (2002) aferiram o lactato 
de gatos saudáveis perante a algumas situações de estresse e demonstraram que ocorre o aumento do lactato apenas nos gatos que fizeram muito esforço físico, com valor máximo de 7,1 $\mathrm{mmol} / \mathrm{L}$, atribuindo essa hiperlactatemia a glicólise gerada devido a anaerobiose causada em um esforço extremo.

No presente estudo o valor médio do lactato foi de $3,4 \pm 0,87 \mathrm{mmol} / \mathrm{L}$, sendo o mínimo de 1,4 mmol / L e o máximo de $6,8 \mathrm{mmol} / \mathrm{L}$. Esses valores são maiores que o valor médio encontrado em cães e do que o valor encontrado em gatos no estudo de Redavid et al (2012). Além disso, diferem do relato no estudo de Rand et al (2002), pois os animais foram manipulados de uma forma menos estressante possível. Apesar de não haver uma correlação estatisticamente significativa entre o maior grau de vasoconstrição periférica com maiores valores de lactato, pode-se perceber uma tendência clínica que une estes parâmetros sob o ponto de vista fisiológico. É possível notar que quanto maior a vasoconstrição apresentada ao exame clínico, maior é o valor de lactato e também mais sinais clínicos de vasoconstrição aparecem (maior TPC e menos borborigmos intestinais). De qualquer forma, é necessário que estas medidas sejam realizadas em uma população maior de animais e em um estudo específico, a fim de encontrar o possível efeito da vasoconstrição sobre os níveis de lactato.

Quando as variáveis que demonstram vasoconstrição periférica foram correlacionadas com os gradientes de temperatura, percebemos que os gradientes de temperatura se alteraram, o TPC aumentou e os borborigmos intestinais diminuíram proporcionalmente ao grau de vasoconstrição periférica. A coloração da mucosa não obteve modificação aparente, estando todos os pacientes normocorados e, por isso, não foi utilizada para a correlação. 


\section{CONCLUSÕES}

Com este estudo podemos concluir que:

Os valores dos gradientes de temperatura e de lactato avaliados na população do estudo poderão ser utilizados para auxiliar o clínico quando for observado variações hemodinâmicas nos pacientes felinos;

Para aplicação desses gradientes devemos considerar as possíveis causas de vasoconstrição que possam influenciar os valores de normalidade, como o estresse à manipulação, pois podem gerar gradientes de temperatura maiores nos gatos saudáveis do que o relatado na literatura para as demais espécies;

A aferição dos gradientes centro-periférico e periférico-ambiental podem ser realizados tanto no membro torácico quanto no pélvico;

Os gradientes de temperatura são semelhantes para machos e fêmeas e podem ser aferidos independentes do resultado de lactato. 


\section{REFERÊNCIAS BIBLIOGRÁFICAS}

AKAMINE, N. et al. Fisiopatologia do choque e da disfunçao de múltiplos órgaos - Cap.3. In: KNOBEL, E. Condutas no Paciente Grave. 3a. ed. Sao Paulo: Atheneu, v. 1, p. 41-60, 2010.

AKATA, T. et al. Reliability of fingertip skin-surface temperature and its related thermal measures as indices of peripheral perfusion in the clinical setting of the operating theatre. Anaesth Intensive Care, p. 32:519-529, 2004.

ALDRICH, J. Global Assessment of the Emergency Patient. Veterinary Small Animal Practice, p. 281-305, 2005.

ALLEN, S. E.; HOLM, J. L. Lactate: physiology and clinical utility. Journal of Veterinary Emergency and Critical Care, 18(2): 123-132, 2008.

ANTONELLI, M. et al. Hemodynamic Monitoring in Shock and Implications For Management. Intensive Care Medicine, 33: 575-590, 2006.

BROWN, S. et al. Guidelines for the identification, evaluation, andd management os systemic hypertension in dog and cats. Journal of Veterinary Internal Medicine, 27: 542 - 558. 2007.

CUNNINGHAM, J.G.: Tratado de Fisiologia Veterinária, 1a Edição, Editora Guanabara Koogan, 1993. 454p.

CURLEY, F.; SMYRNIOS, N. Routinemonitoring of critically ill patients. Journal of Intensive Care Medicine, p. 153-174, 1990.

DAY, M. J .; MACKIN, A.; LITTLEWOOD, J. D. BSAV manual of canine and feline hematology and transfusion medicine. Chapter fifteen. BSVA, 2000. p 263 - 307

FEGEREMAIL, J.; BRAUNE, S. Measurement of skin vasoconstrictor response in healthy subjects. Autonomic Neuroscience: Basic and Clinical, p. 88 - 96, 2005. 
GENDEREN, M.; LIMA, A.; BOMMEL, J. Monitoring peripheral perfusion in critically ill patients at the bedside. In: GENDEREN, M. E. V. Peripheral Perfusion in Relation to Systemic Hemodynamics and its Importance in Critically Ill Patients. Rotterdam: Erasmus Universiteit Rotterdam,. p. 18:273-279, 2012.

HENNING, R.; WEIL, M.; WEINER, F. Blood lactate as prognostic indicator of survival in patients with acute myocardial infarction. Circulatory Shock Journaul, 9: 307-315, 1982.

HOUSE, J.; TIPTON, M. Using skin temperature gradients or skin heat flux measurements to determine thresholds of vasoconstriction and vasodilatation. European Journal of Applied Physiology, p. 88:141-145, 2002.

JOLY, H.; WEIL, M. Temperature of the great toe as an indication of the severity of shock. Circulation, p. 39:131-138, January, 1969.

KOCIBA, G. J. Alterações leucocitárias na doença. In: ETTINGER, S. J.; FELDMAN, E. C. Tratado de medicina interna veterinária: doenças do cão e do gato. Rio de Janeiro: Guanabara Koogan, p. 1941-1956, 2004.

LIMA, A. et al. The prognostic value of the subjective assessment of peripheral perfusion in critically ill patients. Critical Care Medicine, 934 - 938, March, 2009.

LIMA, A.; BAKKER, J. Noninvasive monitoring of peripheral perfusion. Intensive Care Med, p. 1316-1326, 2005.

LIMA, A.; BAKKER, J. Clinical monitoring of peripheral perfusion: there is more to learn. Critical Care, 18:113, 21 Feb 2014.

OSTINI, F. M. et al. O USO DE DROGAS VASOATIVAS EM TERAPIA INTENSIVA. Medicina,. 400-411, jul-set, 1998.

POLI DE FIGUEIREDO, L. F.; SILVA, E.; CORRÊA, T. D. Avaliação hemodinâmica macro e microcirculatória no choque séptico. Revista de Medicina, 84-91, abr-jun 2008. 
RABELO, R. C.; FERRARI, D. Métodos de Avaliação da Perfusão no Paciente Grave. Journal of Latin American Veterinary Emergency and Critical Care Society, p. 134 - 154, 2010 .

RABELO, R. C.; RIBEIRO, C. A. Conceitos de hemodinâmica e microcirculação. In: RABELO, R. C. Emergências de pequenos animais. Rio de Janeiro: Elsevier, p. Rabelo, RC. Emerging Monitoring Techniques. In: Silverstein D., et al. Textbook of Small Animal Emergency Medicine. Wiley Blackwell, Oxford, UK, 2016. Chapter 159, book in progress 25 $-42,2016$.

RAND, J. et al. Acute stress hyperglycemia in cats is associated with struggling and increased concentrations of lactate and norepinephrine. Journal of Veterinary Internal Medicine,16:126$132,2002$.

RÉA-NETO, A. et al. Consenso Brasileiro de Monitorização e Suporte Hemodinâmico - Parte IV: Monitorização da Perfusão Tecidual. Revista Brasileira de Terapia Intensiva, Vol 18, N $^{\circ}$ 2, 154 - 160, Abril - Junho 2006

REDAVID, L. et al. Plasma lactate measurements in healthy cats. Journal of Veterinary Emergency and Critical Care, p. 580-587, 2012.

RUBINSTEIN, E.; SESSLER, D. Skin-surface temperature gradients correlate with fingertip blood flow in humans. Anesthesiology, p. 73:541-545, 1990.

SCHWARTZ, P. J. et al. A Cardiocardiac Sympathovagal Reflex in the Cat. Circulation Research, Vol. XXXII, 215 - 220, February 1973.

TOLEDO JÚNIOR, J. C.; RABELO, R. C. Laboratório de urgências. In: RABELO, R. Emergências de pequenos animais. Rio Janeiro: Elsevier, p. 263, 2012

VILLASEÑOR-MORA，C.; SANCHES-MARIN，F. J.; CALIXTO-CORRERA， S. An indirect skin emissivity measurement in the infrared thermal range throught reflection of a CO2 laser beam. Revista Mexicana de Física, p. 64 - 68, 2009.

VINCENT, J. L.; MORAINE, J. J.; VAN DER LINDEN, P. Toe temperature versus transcutaneous oxygen tension monitoring during acute circulatory failure. Intensive Care Medicine, 64 - 68, march 1988. 
VIVEIROS, J. P.; MEYER, F.; KRUEL, L. F. M. Imersão em água fria para o manejo da hipertermia severa. Revista Brasileira de Medicina do Esporte, Jul/Ago, 609 - 612, 2009.

WEIL, M. H.; HENNING, R. J. New concepts in the diagnosis and fluid treatment of circulatory shock. Thirteenth annual Becton, Dickinson and Company Oscar Schwidetsky Memorial Lecture. Official Journal of the International Anesthesia Research Society, 124 132, March/April 1979. 